\title{
Entrevista cualitativa y la investigación en educación de adultos
}

\author{
Rafael M. Hernández-Carrera* \\ Rodrigo Matos de Souza** \\ Elizeu Clementino de Souza***
}

\begin{abstract}
Resumen
El presente artículo se centra en los aspectos teóricos y metodológicos en los que se basan los trabajos que utilizan la entrevista como instrumento de recogida de datos empíricos en las investigaciones de corte cualitativo. Desde una mirada epistemológica, este trabajo hace un recorrido por el camino de la utilización de las entrevistas en el marco de las Ciencias Humanas, describiendo la educación de adultos como espacio de cuestionamiento y territorialidad de estos instrumentos. El método de producción de este texto lo podemos enmarcar en el bricolage, por otra parte, respecto a la perspectiva de análisis y problematización de estos instrumentos, los encuadramos en la Educación de Adultos como realidad socioeducativa transformadora de los individuos y los colectivos.

Palabras-clave: Educación de Adultos; Entrevistas; Métodos Cualitativos
\end{abstract}

\section{Entrevista qualitativa e a pesquisa em educação de adultos}

\section{Resumo}

O presente artigo ocupa-se dos aspectos teórico-metodológicos nos quais se fundamentam os estudos que utilizam a entrevista como instrumento de recolha de dados nas pesquisas de natureza qualitativa. A partir de um enfoque epistemológico, discute o uso das entrevistas no horizonte das Ciências Humanas, descrevendo a educação de adultos como espaço de questionamento e territorialização deste dispositivo de pesquisa-formação. O método de produção deste texto foi a Bicolage, e, no que diz respeito à perspectiva de análise e problematização dos instrumentos discutidos, localizamos a Educação de Adultos como realidade socioeducativa transformadora dos indivíduos e dos coletivos humanos.

Palavras-Chave: Educação de Adultos; Entrevistas; Métodos Qualitativos

\section{Qualitative interview and research in adult education}

\begin{abstract}
Keywords: Adult Education; interviews; Qualitative methods

\section{La investigación cualitativa}

En primer lugar nos gustaría significar que la investigación cualitativa abarca distintas fases no conteniendo un inicio y un fin claramente definido. Estas fases se entremezclan continuamente produciendo una espiral reflexiva continua. Aun, se puede decir que la idea de calidad asociada a la investigación es muy antigua y nos remitiría a una mirada retrospectiva, probablemente sin necesidad, para ubicar la investigación cualitativa en un horizonte clásico, por esto mismo, aquí, tratamos de

*Endereço Eletrônico: rmhercar@upo.es

${ }^{* *}$ Endereço Eletrônico: rodrigomatos28@ @otmail.com

*** Endereço Eletrônico: esclementino@uol.com.br
\end{abstract}

This article concerns the theoretical and methodological aspects of which are grounded studies using the interview as data collection instrument in qualitative research. From an epistemological approach, it discusses the use of interviews on the horizon of Human Sciences, describing the adult education as a space of questionning and territorialization of these research-formation device. The production method of this text was Bricolage, and, with regard to the prospect of analysis and questioning of the instruments discussed, we placed adult education as a transforming social and educational reality of individuals and human collectives.

elegir un punto de partida que enmarque el ascenso de la calidad como antítesis de la cantidad en el paradigma de las ciencias humanas.

Bajo la denominación de investigación cualitativa se incluyen varios tipos de investigación que pueden diferenciarse, entre otras cosas, por los supuestos teóricos de los que parten. Así, Flick (2012) sostiene que estas posiciones teóricas se pueden resumir en tres que son el interaccionismo simbólico, la etnometodología y el estructuralismo ${ }^{1}$. El primero se interesa por los significados subjetivos que los individuos dan a los objetos. Su 
interés se focaliza en los procesos de interacción y por tanto la investigación con raíces en él se basan en el simbolismo de las acciones sociales. Por su parte la etnometodología pone su foco en las cuestiones cotidianas y en el modo en que éstas se generan, es decir, se interesa por cómo las personas producen los procesos de interacción social. Por último, el modelo estructuralista-psicoanalítico se basa en los procesos inconscientes, tanto desde el punto de vista psicológico como social.

\section{El Interaccionismo Simbólico}

Este término lo introdujo Herbert Blumer a finales de los años treinta del siglo XX, apoyándose en las ideas de William James y John Dewey, entre otros. Según él, el ser humano sitúa sus actos hacia los objetos en función de lo que éstos significan para él. El origen de ese significado es un producto social que procede de y a través de las actividades de los individuos al interactuar. La utilización del significado por el individuo se produce a través de un proceso de interpretación propia que supone autointeracción y manipulación de significados. En este sentido, "las diferentes maneras en que los individuos revisten de significados los objetos, los acontecimientos, las experiencias, etc., forman el punto de partida central para la investigación. La reconstrucción de estos puntos de vista subjetivos se convierte en el instrumento para analizar los mundos sociales" (FLICK, 2012, p. 32). También cita este autor en relación con este tema el denominado Teorema de Thomas: "Cuando una persona define una situación como real, esta situación es real en sus consecuencias" (FLICK, 2004, p. 34).

Este principio, como señala Flick (2012) en su discusión, conlleva al punto central de esta perspectiva metodológica, que es que el investigador debe intentar ver el mundo desde la perspectiva de los investigados ${ }^{2}$. En definitiva, este supuesto teórico está presente en el estudio ya que el investigador se centra en conocer el punto de vista de los entrevistados respecto al objeto de estudio.

\section{Etnometodología}

Los estudios de la Etnometodología están ubicados en lo que se suele identificar como microsociología enmarcada por el enfoque interpretativo y por la adopción de métodos producidos por inexpertos y por los grupo populares a partir de lo que utilizan, en su cotidianidad, para dar sentido a lo que dice o producen dentro de su contexto. Es un esfuerzo de razonamiento de las prácticas de los grupos, en su naturaleza descriptiva, lo que significa que tiene un rasgo no teórico o metódico de antemano y su fundación va en contra a la "sociología profesional" que intenta rellenar de sentido ajeno las práctica de los otros (OCÁDIZ, 2016; FLICK, 2012).

\section{Fenomenología y Hermenéutica}

También, consideramos que es importante referir aquí someramente dos corrientes filosóficas que están presentes a lo largo del artículo y que de un modo u otro la impregnan, la Fenomenología y la Hermenéutica. Etimológicamente, Fenomenología proviene del griego antiguo Phaeinomenon "lo que se presenta o lo que se muestra" y logos "razón, principio, inteligencia", y plantea que hay que analizar los fenómenos como aparecen. La máxima de este movimiento filosófico introducido por Edmund Husserl (1982) es plegarse a las cosas mismas, es decir, ser fiel a lo que realmente se presenta o a lo que se muestra en la consciencia.

Según Kvale (2011) esta corriente describe y analiza la consciencia y se centra en lo que él denomina "el mundo de la vida", tratando de conocer y centrarse en la experiencia de los sujetos. Pone un gran énfasis en las descripciones precisas y trata de poner entre paréntesis los conocimientos previos. Por su parte, Vallés hace la siguiente reflexión respecto a la fenomenología en el marco de la investigación cualitativa, en general, y a las entrevistas, en particular:

El sustrato fenomenológico estaría en el intento de interpretación de lo dicho o hecho por la gente desde su punto de vista. Más aún, en lo dicho o hecho por la gente estaría la huella de su visión del mundo. De ahí el interés en la observación y en el registro del lenguaje natural (VALLÉS, 2009, p. 41).

En definitiva, la Fenomenología se ocupa de las vivencias y de pensar sobre el propio conocimiento, o dicho de otro modo se ocupa de conocer el conocimiento.

La Hermenéutica surge como disciplina filosófica y metodología para la comprensión de las Ciencias Humanas recientemente, su origen remonta al trabajo desarrollado por el teólogo e filósofo Schleiermacher -existen menciones a otros 
emprendimientos interpretativos, pero estos estuvieron limitados a los estudios de las lenguas clásicas, como nos acuerda Schmidt (2012) y el propio Schleiermacher (2001). Diferente de las tradiciones criticadas por el exegeta alemán, la comprensión estaría más allá del texto, y para que la interpretación exista se debe dedicarse a saber cómo la interpretación se da. En este sentido, la búsqueda de la Hermenéutica, en sentido general, es proveer las razones de la interpretación y no solamente decir cómo interpretar a uno u otro texto.

Lo que se busca con esa técnica ${ }^{2}$ es el restablecimiento de los sentidos - Ricoeur (2011) preferirá el uso de la terminología significación exteriores al texto impreso, que en el contexto schleiermacheriano significa la reconstrucción de los eventos objetivos y subjetivos de un discurso.

Estas dos corrientes, la Fenomenología y la Hermenéutica, están presentes de uno u otro modo en la esencia de este artículo y hemos considerado presentar este abordaje sobre las mismas.

\section{Características de la investigación cualitativa}

Flick (2012) indica que existen ciertas características que identifican la investigación cualitativa. Por una parte, los investigadores centran su interés en acceder a las experiencias y los datos en su entorno natural de manera que pueda existir un espacio para que se expresen las peculiaridades de las mismas. Otro rasgo fundamental es que este tipo de investigación no tiene en un principio un concepto claro de lo que se estudia ni una hipótesis que después se pueda validar. Los conceptos y las hipótesis se van formulando a lo largo de la propia investigación. Los métodos y las teorías que se aplican al objeto de estudio deben adaptarse al mismo y si no es así se reformulan, se adaptan o se desarrollan nuevos métodos o enfoques. Así, el investigador es una parte fundamental del proceso de investigación no ya solo aportando experiencias de campo sino participando con su propia reflexividad. A continuación recogemos una interesante aportación de Flick sobre esta cuestión:
A diferencia de la investigación cuantitativa, los métodos cualitativos toman la comunicación del investigador con el campo y sus miembros como una parte explícita de la producción de conocimiento, en lugar de excluirla lo más posible como una variable parcialmente responsable. Las subjetividades del investigador y de aquéllos a los que se estudia son parte del proceso de investigación. Las reflexiones de los investigadores sobre sus acciones y observaciones en el campo, sus impresiones, accesos de irritación, sentimientos, etc., se convierten en datos de propio derecho, formando parte de la interpretación, y se documentan en diarios de investigación o protocolos de contexto (FLICK, 2012, p. 20).

Según Denzin y Lincoln (2012) existen 5 fases interconectadas entre sí que definen el proceso de investigación cualitativa. Estas son el propio investigador, los paradigmas en los que basa su estudio, las estrategias de investigación, los métodos de recolección y análisis del material empírico y la interpretación. A continuación desarrollamos esquemáticamente como entienden estos autores y estas fases.

La primera de ellas está centrada en el investigador; este tiene una biografía personal que le lleva a aproximarse al objeto de estudio desde sus distintas dimensiones como ser humano: su cultura, su género, su raza, su clase, las experiencias vividas etc. La segunda hace referencia al modo en el que el investigador se aproxima al objeto de estudio, ya que este estará desde un principio condicionado por el paradigma desde el que lo haga. En términos generales un paradigma lo podemos definir como un marco interpretativo o como una red que contiene las premisas epistemológicas, ontológicas y metodológicas desde las que se abordará la investigación. Según estos autores existen, en términos generales, cuatro paradigmas fundamentales en la investigación cualitativa que son el positivista, el constructivista - interpretativo, el crítico y el feminista ${ }^{3}$. A continuación se presenta una tabla tomada de Denzin y Lincoln (2012, p. 87) en la que se esquematizan los distintos paradigmas. 
Tabla 1. Paradigmas

\begin{tabular}{|c|c|c|c|}
\hline PARADIGMA/TEORIA & CRITERIOS & $\begin{array}{l}\text { FORMA DE } \\
\text { TEORÍA }\end{array}$ & $\begin{array}{c}\text { TIPO DE } \\
\text { NARRATIVA }\end{array}$ \\
\hline Positivista / pospositivista & Validez interna y externa & $\begin{array}{l}\text { Fundamentada, lógico- } \\
\text { deductiva }\end{array}$ & Informe cientifico \\
\hline $\begin{array}{l}\text { Constructivista / } \\
\text { interpretativo }\end{array}$ & $\begin{array}{l}\text { Confiabilidad, credibilidad, } \\
\text { transferibilidad, confirmabilidad }\end{array}$ & Sustantiva-formal & $\begin{array}{l}\text { Estudio interpretativo de } \\
\text { casos, ficción etnográfica }\end{array}$ \\
\hline Feminista & $\begin{array}{l}\text { Afrocéntrico, experiencia vital, } \\
\text { diálogo, cuidado, responsabilidad, } \\
\text { raza, clase, género, reflexibilidad, } \\
\text { praxis, emoción, fundamentación } \\
\text { concreta }\end{array}$ & $\begin{array}{l}\text { Crítica, basada en el } \\
\text { punto de vista }\end{array}$ & $\begin{array}{l}\text { Ensayos, relatos, escritura } \\
\text { experimental }\end{array}$ \\
\hline Ėtnico las, & $\begin{array}{l}\text { Afrocéntrico, experiencia vital, } \\
\text { diálogo, cuidado, responsabilidad, } \\
\text { raza, género, clase }\end{array}$ & $\begin{array}{l}\text { Crítica, histórica, basada } \\
\text { en un punto de vista }\end{array}$ & Ensayos, fábudramas \\
\hline Marxista & $\begin{array}{l}\text { Teoría emancipatoria, falseabilidad } \\
\text { dialógica, raza, clase, género }\end{array}$ & $\begin{array}{l}\text { Crítica, histórica, } \\
\text { económica }\end{array}$ & $\begin{array}{l}\text { Análisis histórico, } \\
\text { económico y sociocultural }\end{array}$ \\
\hline Estudios culturales & $\begin{array}{l}\text { Prácticas culturales, praxis, textos } \\
\text { sociales, subjetividades }\end{array}$ & Critica social & $\begin{array}{l}\text { Teoría cultural como } \\
\text { crítica }\end{array}$ \\
\hline Teoría queer & Reflexibilidad, deconstrucción & $\begin{array}{l}\text { Crítica social, análisis } \\
\text { histórico }\end{array}$ & $\begin{array}{l}\text { Teoría como crítica, } \\
\text { autobiografia }\end{array}$ \\
\hline
\end{tabular}

Fuente: Denzin y Lincoln (2012)

La tercera fase hace referencia a la estrategia de investigación, la cual comienza con el diseño de la misma. Según estos autores el diseño contiene una serie de pautas flexibles que conectan por una parte los paradigmas teóricos con las estrategias, y por otra, con los métodos para obtener material empírico en el cual basarse en la fase de interpretación.

La siguiente fase hace referencia a los métodos de recolección y análisis del material empírico. Existen diversos modos o técnicas de recoger este material entre los que podemos destacar la entrevista, la observación, el análisis de documentos, de materiales visuales y la propia experiencia personal.

La quinta fase es la interpretación. En el caso de la investigación cualitativa esta fase puede asimilarse a un proceso de construcción. El investigador parte de sus notas o diario de campo para producir después un texto de investigación en base a ellos. Posteriormente ese texto de investigación es reescrito como un documento interpretativo funcional que contiene un primer intento de dar significado a lo que estudió. La última cuestión es la redacción del texto / informe final que será el que se aporte a la comunidad científica o simplemente a los interesados en el campo de estudio.

De cualquier modo, un abordaje cualitativo no está basado en metodologías deductivas tradicionales, donde se parte de un modelo teórico determinado y de unas hipótesis que tratan de validarse o de negarse a través los datos empíricos extraídos de las fases de obtención de datos. En palabras de Goetz y LeCompte (1988) una investigación que parta de una técnica deductiva empieza con una teoría determinada desarrollando definiciones y conceptos que aplica empíricamente a algún conjunto de datos. En otras palabras, pretende encontrar datos que corroboren la teoría. Por el contrario la investigación inductiva comienza con la fase de recogida de datos empíricos a través de algún sistema de recogida de información, como pueden ser la observación o las entrevistas, y a partir de los hallazgos construye las categorías y proposiciones teóricas.

Otra cuestión importante desde el punto de vista epistemológico es el concepto de Verstehen introducido por Weber, término alemán que significa comprender, entender, ver con la inteligencia, una comprensión empática o una habilidad para reproducir en la propia mente los sentimientos, motivos y pensamientos detrás de las acciones de otros, en términos de Vallés (2009). 
Desde este punto de vista, la investigación cualitativa trata de comprender un fenómeno concreto, para lo cual se va a apoyar en la visión que del mismo tiene un sujeto o sujetos diferentes.

\section{La investigación a través de entrevistas}

Superada la época en la que el abordaje desde el paradigma positivista era el que confería soporte teórico y metodológico a las investigaciones sociales, los múltiples cambios de orientación paradigmática en las metodologías cualitativa y cuantitativa han derrumbado las murallas que existían entre ellas y permitido una mayor complejidad en el abordaje de los métodos; esto contribuyó a un ensemble de las producciones científicas en las Ciencias Sociales y Humanas, puesto que ahora se aceptan sin grandes traumas que un estudio se proponga cualitativo y cuantitativo, en muchos casos, sin causar gran malestar. Esta crisis paradigmática (SANTOS, 2006) también ocurrió en el caso de las entrevistas, en el pasado esquemáticas, que ganaron otros contornos y otras posibilidades materiales.

Según Kvale (2011) actualmente la investigación en Ciencias Sociales suele combinar las entrevistas con otros métodos. Un ejemplo claro de ello es la investigación de Bourdieu (2007) sobre la marginación en el sistema educativo y la marginación social, donde combinaba métodos cualitativos y cuantitativos.

Llegados a este punto consideramos importante detenernos brevemente en el apartado de las entrevistas. Presentaremos un recorrido por esta técnica de recogida de información tanto desde el punto de vista histórico como tipológico y metodológico. Describiremos la entrevista como una de las técnicas más importantes de recogida de datos en la investigación cualitativa. También, nos referiremos a las técnicas de discusión grupal como pueden ser el grupo de discusión o el debate.

En primer lugar analizamos el origen etimológico de la palabra ya que de él podemos extraer una primera información. El vocablo "entrevista" está compuesto por entre (del latín inter) y vista (derivada del verbo latino videre, que significa ver). Es decir, entrevista puede significar ver algo entre dos, aproximarnos al objeto de estudio tomando como referencia la visión de dos individuos en vez de uno solo. El conocimiento se va a construir a partir de la interacción entre el entrevistador y el entrevistado. No se trata, únicamente, de hacer preguntas a una persona que posee el conocimiento para que nos lo transmita sino que se le va a pedir, también, procesamiento y elaboración de las respuestas. En este sentido, la entrevista tiene la finalidad de tomar el conocimiento desde el punto de vista del entrevistado. Es, en cierto modo, un tipo de interacción conversacional con rasgos particulares que necesitan ser entendidos; es un tipo de interacción conversacional cara a cara (WENGRAF, 2012). En este punto, retomamos la reflexión de Atkinson en relación con la naturaleza de las entrevistas. Nos dice que la entrevista no es una conversación, y aunque mantenga alguna identificación con esta práctica social, la posición de quién entrevista debe ser la de escucha y permanecer en segundo plano (ATKINSON, 1998)

Según Kvale (2011) las conversaciones o los diálogos son una forma ancestral de obtener conocimiento. Comenta este autor como en la antigua Grecia Tucídides entrevistaba a los guerreros que regresaban de las guerras del Peloponeso para escribir su obra "Historia de la guerra del Peloponeso", guerras que se extendieron durante veintisiete años a lo largo del siglo $\mathrm{V}$ a.C. Abajo recogemos un fragmento de esta obra en la que podemos apreciar cómo su autor no se dejaba llevar por las primeras impresiones, haciendo uso no sólo de la observación en el campo de batalla, sino que también se apoyaba en las versiones que le narraban los distintos protagonistas de la misma.

En cuanto a los hechos acaecidos en el curso de la guerra, he considerado que no era conveniente relatarlos a partir de la primera información que caía en mis manos, ni como a mí me parecía, sino escribiendo sobre aquello que yo mismo he presenciado o que, cuando otros me han informado, he investigado caso por caso, con toda la exactitud posible. La investigación ha sido laboriosa porque los testigos no han dado las mismas versiones de los mismos hechos, sino según las simpatías por unos o por otros o según la memoria de cada uno (TUCÍDIDES, 1989).

Continuando con la antigua Grecia, es sobradamente conocido el conocimiento filosófico desarrollado por Sócrates a partir de los diálogos. Si bien fue Platón quien introdujera el género filosófico - literario del diálogo socrático, otros clásicos como Jenofonte, Aristóteles, Cicerón, Séneca y posteriormente Galileo Galilei, Voltaire, Rousseau, Diderot o Fray Luis de León compusieron textos filosóficos con inspiración en 
este género.

Otra importante aportación a la técnica de la entrevista es la que hace la psicología desde un punto de vista científico o terapéutico. Así, Sigmund Freud basa gran parte de la Teoría Psicoanalítica en las entrevistas terapéuticas con sus pacientes. Por su parte Piaget basó también gran parte de sus teorías en entrevistas a niños y en la observación de los mismos. Otros Psicólogos como Carl Rogers y su psicoterapia centrada en el cliente, hicieron de la entrevista su base fundamental de trabajo. En este sentido, Rogers introduce la palabra "cliente" en vez de "paciente" ya que esta acepción le confiere responsabilidad y libertad sobre el proceso terapéutico como un elemento activo, matiz que no se recoge en el término paciente que hace más referencia a "tener paciencia" con el problema, convirtiéndolo en un sujeto pasivo.

Por último mencionamos aquí la aportación que han hecho a la técnica de la entrevista disciplinas como el periodismo o la selección de personal, las cuáles hacen de ella la principal fuente de trabajo e información.

\section{Modelos de representación}

En primer lugar queremos poner de manifiesto que existen varios modelos para trabajar con entrevistas que van, desde una concepción más positivista basada en la recogida de datos como hechos, hasta una concepción más postmoderna que percibe la investigación con entrevistas como un proceso de construcción y narrativo (SCHÜTZE, 1992; JOVCHELOVITCH, S.; BAUER, M.W, 2002; WELLER; ZARDO, 2013; MUYLAERT et al., 2014). En otras palabras, en la investigación social distinguimos entre recopilación de datos e interpretación de datos $\mathrm{y}$, dependiendo de cómo se relacionen estos dos conceptos, hablaremos de un modelo hipotético - inductivo o de otro hipotético deductivo (WENGRAF, 2012).

Según este autor en el modelo del sentido común, el hipotético - inductivo, el investigador recopila todos los hechos relevantes tras lo cual los examina, los analiza y los estudia para ver qué teoría le sugieren los mismos. Los hechos son los que sugieren $\mathrm{o}$, incluso, dictan la teorización. El modelo contrario es el hipotético - deductivo, el cual plantea que la investigación debe partir de un cuerpo teórico y a partir de ahí decidir qué hechos o qué datos hay que recopilar. Es a partir de este cuerpo teórico cuando el investigador genera una hipótesis sobre la que trabajar.

Al tratarse esta investigación de un estudio basado en entrevistas semiestructuradas, no podemos afirmar que vaya a ser puramente hipotético -inductivo o que no tenga algo de hipotético - deductivo. A continuación adaptamos una ilustración de Wengraf (2012) en la que se recoge el espectro que va desde las entrevistas fuertemente estructuradas a las no estructuradas y lo fusionamos con las metáforas del investigador como viajero y como minero de Kvale.

Ilustración 1 - Espectro desde entrevistas no estructuradas hasta entrevistas fuertemente estructuradas y relaciones de las fases en el desarrollo de Teoría

\begin{abstract}
Construcción del modelo
Comprobación del modelo

Construcción de la teoría

Comprobación de la teoría
\end{abstract}

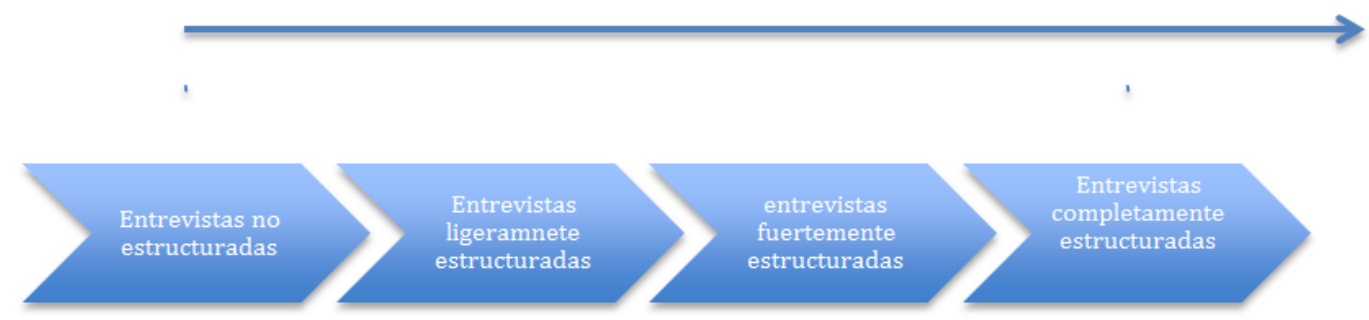

Investigador como viajero

Investigador como minero

Adaptación de: Wengraf (2012, p. 61). 
Tipos de entrevistas

Una primera diferenciación entre entrevistas es la que hace Kvale (2011) en función de los sujetos entrevistados. Así, distingue entre las entrevistas realizadas a sujetos de distintas culturas, lo cual supone una forma especial de acercarse a ellos ya que cada cultura tiene normas distintas en la forma de interaccionar con personas extrañas, sobre todo en cuestiones relativas a la franqueza, la iniciativa, las formas de preguntar, etc. (y tales entrevistas por su respeto a las dinámicas culturales pueden servir bien a los investigadores de la Educación de Adultos); las entrevistas con niños, las cuales permiten a éstos expresar sus experiencias y la manera en la que perciben y comprenden su mundo. En este sentido, la experiencia de Piaget con niños en relación a la percepción de conceptos físicos y a la forma en la que ellos comprenden la realidad y las cuestiones morales han configurado en gran medida las concepciones que actualmente se tienen sobre el pensamiento infantil.

González-Monteagudo (2010) hace una distinción de enfoques apoyándose en Miller en relación con la investigación biográfica y las entrevistas.

[...] Miller distingue y sintetiza tres enfoques: realista, neopositivista y narrativo. El enfoque realista es inductivo y defiende un tipo de entrevista no-directivo o semi-directivo. Este enfoque tiene una fuerte relación con la grounded theory (teoría fundamentada). El punto de vista de los actores sociales se considera importante, pues supone un elemento importante de la realidad social. Para favorecer una mejor comprensión social, el enfoque realista trabaja con el concepto de saturación, es decir, con la idea de que el estudio de un mayor número de casos permitirá una generalización débil, a partir del momento en que los nuevos casos aporten pocas novedades respecto de los casos ya documentados (GONZÁLEZMONTEAGUDO, 2010, p. 23).

$\mathrm{Si}$ atendemos a las formas de entrevistas, podemos encontrar un amplio repertorio de estas de entre las cuales habrá que dilucidar cuál sería el más apropiado para cada investigación. A continuación referimos algunas de ellas y exponemos otras que consideramos de interés.

\section{Entrevista narrativa}

Las entrevistas narrativas ponen su foco en las historias que los sujetos cuentan de su propia vida, o de una parte de esta, teniendo en cuenta su propio punto de vista. Se centran en las tramas y estructuras de los relatos pudiendo surgir bien espontáneamente bien provocadas por el entrevistador (KVALE, 2011). En este sentido, las narraciones contadas por los sujetos son, también, datos susceptibles de ser analizados empíricamente. Se utiliza, principalmente en el ámbito de la investigación biográfica.

Normalmente se comienza este tipo de entrevistas con una pregunta inicial generadora de la narración del entrevistado con la finalidad de estimular y motivar a éste a contar la historia.

En la entrevista narrativa, se pide al informante
que presente la historia de un área de interés, en
la que participó el entrevistado, en una narración
improvisada... La tarea del entrevistador es
hacer que el informante cuente la historia del
área de interés en cuestión como un relato
coherente de todos los acontecimientos
relevantes desde su principio hasta su final
(HERMANNS, 1995, p. 111).

Las entrevistas narrativas están muy presentes en la investigación con entrevistas, especialmente en el ámbito de la investigación biográfica. Pueden centrarse en un episodio concreto de la vida de una persona, denominándose historia breve, como puede ser de algún modo en el caso de nuestra investigación cuando se pide información a los entrevistados sobre su vida profesional solicitándoles que nos cuenten su bagaje o historia laboral. También pueden centrarse en conocer el curso de la vida del entrevistado, denominándose entonces historia de vida o entrevista biográfica. En ella la persona nos cuenta desde su propia perspectiva su vida, su historia vital, erigiéndose en el protagonista de la obra. Por último, cuando esta historia va más allá de la vida del individuo y pasa a formar parte de uno de los relatos a través del que se construye la historia de una comunidad, se llama entonces historia oral (KVALE, 2011). En palabras de GonzálezMonteagudo, "La historia oral representa no solo un nuevo enfoque técnico y metodológico de la historia sino una transformación de paradigma y perspectiva" (GONZÁLEZ-MONTEAGUDO, 2010, p. 29). Así el movimiento de la historia oral ha favorecido el conocimiento de la metodología y los enfoques teóricos de la investigación a través de fuentes orales y biográficas (GONZÁLEZMONTEAGUDO, 2010). 
En teorizar sobre la entrevista narrativa, como una de las posibilidades de recolección de datos en la investigación biográfica, DeloryMomberger discute los aspectos de este procedimiento, destacando el papel de la subjetividad, de las experiencias constructivas de la individualidad y de los procesos de individuación, mediante el ejercicio dialógico de escucha de las disposiciones de la "exterioridad e interioridad personal" (2012, p. 526) de los tiempos y espacios de las biografías individuales y colectivas. De esta forma, entrevistar tiene que ver con dimensiones heurísticas, pues implica en ponerse a oír las historias narradas, visto que:

[...] La entrevista de investigación biográfica establece así un doble inversión de investigación, un doble espacio heurístico que acciona sobre cada una de los involucrados: el espacio del entrevistado en la posición de entrevistador de sí mismo; el espacio del entrevistador, el cuál objeto proprio es producir las condiciones de entender el trabajo del entrevistado sobre sí mismo (DELORYMOMBERGER, 2012, p. 527).

Las disposiciones construidas en un proceso de entrevista abren muchas posibilidades de sentido, formación, entendimiento y huellas biográficas de vida entre entrevistador y entrevistado, frente al intercambio de experiencias de vida y recorridos biográficos. Para Souza (2011), las investigaciones dirigidas por entrevistas narrativas contribuyen para la superación de la racionalidad técnica como principio único y modelo de formación. También porque la investigación con entrevistas narrativas funcionan en una perspectiva colaborativa, a la medida en que quién narra y reflexiona sobre su recorrido abre posibilidades de teorización de su propia experiencias y amplía su formación a través de la investigación y formación de sí. Por otra parte, el trabajo con las entrevistas narrativas remite las alumnas y alumnos en proceso de formación a interrogar sus rutas de desarrollo personal y profesional, mediante la escucha/lectura de la narración del otro. La perspectiva colaborativa de investigación con entrevistas narrativas en el territorio de la formación supone aprendizajes y teorizaciones sobre las prácticas docentes pues provocan experiencias narrativas como posibilidad de comprensión y ampliación de los sentidos de los recorridos de formación y de la propia historia de las diferentes personas vinculadas a un proyecto de formación ${ }^{4}$.

\section{Entrevista centrada en el problema}

La entrevista centrada en el problema fue introducida por Witzel en la década de los 80 del siglo XX. Se vale de la incorporación de una guía de entrevistas que introduce preguntas y cuestiones que estimulan al entrevistado a la narración, a contar algo sobre un problema concreto. En palabras de Flick,

Esta entrevista se caracteriza por tres criterios principales: centrarse en el problema, es decir, la orientación del investigador hacia un problema social pertinente; la orientación al objeto, es decir, que los métodos se desarrollan o modifican con respecto a un objeto de investigación $\mathrm{y}$, por último, la orientación al proceso en el proceso de investigación y en la manera de comprender el objeto de investigación (FLICK, 2012, p. 100).

Dicho de otro modo, esta modalidad de entrevista parte de una guía de entrevista que sirve para dar apoyo al hilo narrativo que desarrolla el entrevistado. También, esta guía es de gran ayuda para dar un giro a la entrevista cuando ésta empieza a discurrir por derroteros que no están muy acordes con el problema de investigación o cuando se llega a momentos de estancamiento en la misma. El entrevistador tiene que decidir cuándo introduce estas preguntas concretas que están centradas en el problema objeto de estudio. Witzel (1985) menciona cuatro estrategias comunicativas principales en la entrevista centrada en el problema: la entrada en conversación, la incitación general, la incitación específica y las preguntas ad hoc.

El interés de esta metodología de entrevista radica en el punto de vista subjetivo de los entrevistados. Con este tipo de entrevistas, la investigación se basa en un modelo de proceso teniendo como finalidad desarrollar teorías; igualmente, la selección de entrevistados debe llevarse a cabo poco a poco y trata de enfocar el problema desde el punto de vista de éstos. Esta cuestión encaja plenamente con metodologías de análisis e interpretación centrados en la codificación, categorización y en el análisis cualitativo de contenidos (FLICK, 2012).

\section{La entrevista a élites y a expertos}

En la entrevista a expertos, también denominada según Dexter como entrevista especializada o a élites (DEXTER, 1970), el 
entrevistado tiene más importancia como individuo perteneciente a un colectivo concreto que como persona individual. Dexter publicó en 1970 un trabajo titulado Elite and Specialized Interviewing, para el que entrevistó a directivos de asociaciones, a políticos, senadores y diversos tipos de personajes públicos.

En este tipo de entrevistas, lo que le confiere interés al entrevistado es el hecho de ser un conocedor experto de una materia o problema así como pertenecer a algún grupo o a alguna élite que tiene una percepción concreta sobre un tema determinado. Aquí, el término élite no necesariamente debe asociarse a personas relevantes de la vida pública (políticos, personas relevantes del mundo de las finanzas o profesiones de prestigio), sino que hace referencia a personas con la calidad de expertos en un tema o sencillamente bien informados. Este es el caso de la totalidad de nuestros entrevistados, los cuales han sido entrevistados en su calidad de personas expertas en el ámbito de la formación en el entorno de las asociaciones empresariales: Presidentes de Asociaciones Empresariales, Directores, Responsables y Técnicos de Formación, Secretarios de Formación de Sindicatos, etc., en definitiva, expertos y personas bien informadas.

La mayor parte de las ideas de Dexter sobre las entrevistas de élites, reconoce el autor, provienen más de la antropología y los antropólogos que de cualquier otro colectivo profesional. Este autor comenta en relación al término élite que éste no le gusta por sus connotaciones de superioridad pero que aún no ha encontrado un término mejor para definir a personas en puestos importantes que requieren un tratamiento de entrevista especial en relación a la importancia de su puesto. De hecho hoy en día son muchas las personas que pueden estar muy bien informadas sobre un tema en particular, por ejemplo, un trabajador que haya formado parte de la historia de la constitución de un sindicato o con una implicación política en este tipo de movimientos, puede ser entrevistado bajo esta modalidad de entrevistas ya que nadie estaría mejor informado que él de cara a mantener una entrevista sobre la cuestión, siendo una autoridad en el tema (DEXTER, 1970).

Una entrevista de élite podría, también definirse como

Una entrevista con cualquier entrevistado que en términos de las intenciones del entrevistador recibe un tratamiento especial, no estandarizado. Por tratamiento especial no estandarizado se entiende poner hincapié en la definición de la situación por parte del entrevistado, animarle a estructurar la situación según su criterio y permitir a éste introducir una considerable y extensa cantidad de nociones sobre lo que se considera relevante en vez de plegarse a lo que el entrevistador considera relevante (RIESMAN Apud DEXTER, 1970, p. 5).

Dicho de otro modo en las entrevistas estandarizadas $y$ en muchas otras entrevistas aparentemente no estandarizadas (como puede ser la entrevista focalizada de Merton en su estilo más puro) el entrevistador define la pregunta y el problema buscando respuestas sólo dentro de los límites programados en sus suposiciones. Sin embargo, en la entrevista a élites el entrevistador se muestra más atento y condescendiente, y a veces ansioso, por permitir al entrevistado mostrarle cuál es el problema, la pregunta o la situación real. Esta aproximación sobre la entrevista se ha llevado a cabo mucho más a menudo en personas que ocupan una posición destacada, influyente, o simplemente personas bien informadas, que en las personas llanas o comunes. De algún modo, muchas personas pertenecientes al primer grupo no suelen estar dispuestos a aceptar los presupuestos de los que el investigador parte, insistiendo en explicarle como ven ellos la situación, y cuáles son los problemas reales según su punto de vista (DEXTER, 1970).

En este tipo de entrevistas cobra mayor importancia y adquiere un carácter más fuerte, si cabe, la guía de entrevistas ya que ella nos permite salirnos de situaciones improductivas en las que las entrevistas pueden llegar a caer. En este sentido son más directivas, si bien en nuestras entrevistas hemos tratado de dejar hueco para las narraciones y para que los sujetos se extendiesen en temas que, aun no estando en la guía de entrevistas, entendíamos que estaban relacionadas con el problema de investigación. El modo de conducir estas entrevistas depende en gran medida de la personalidad y de las habilidades del entrevistador así como de la actitud y la orientación de entrevistado. En cierto modo, es ampliamente asumido que los entrevistados les están haciendo un favor a los entrevistadores accediendo a la entrevista por lo que éstos suelen mostrar cierta deferencia y cortesía a aquéllos.

Otra característica de las entrevistas a élites es que mientras en las entrevistas estandarizadas las desviaciones son frecuentemente tratadas estadísticamente (ej. desviación típica), en las entrevistas a élites, una desviación, una excepción o una explicación poco frecuente pude sugerir una 
revisión, una reinterpretación o una nueva aproximación al objeto de estudio (DEXTER, 1970).

En esta modalidad de entrevistas, también, es de suma importancia que el entrevistado perciba al entrevistador como alguien que conoce bien la materia sobre la que están departiendo de manera que se sienta estimulado a narrar y abundar en las respuestas.

La necesidad de que el entrevistador deje claro en la entrevista que está familiarizado también con este asunto es, en general, una condición para realizarla con éxito. La interpretación de entrevistas a expertos pretende sobre todo analizar y comparar el contenido del experto (FLICK, 2012, p. 105).

Finalmente, comenta Dexter, una vez que la entrevista comienza, el entrevistado obtiene algo de este proceso que le estimula a comunicarse. Probablemente el mayor beneficio que los entrevistados reciben, razón por la cual suelen disfrutar de la entrevista, es la oportunidad de enseñar y de contarle a la gente algo que ellos consideran importante (DEXTER, 1970).

\section{La entrevista en la Educación de adultos: posibilidades $\mathbf{y}$ aberturas}

La Educación de Adultos ${ }^{5}$ es un fenómeno global, diverso, enmarcado por diferentes condiciones de llegada de estos adultos a esta modalidad educativa. A veces como egresado, otras como discapacitado, otras como excluido del sistema, otras aun como recién llegado a una comunidad o sociedad como desplazado, inmigrante o refugiado. Los adultos encuentran en la idea de educarse una posibilidad potenciadora de su condición humana y transformadora de sus vidas para llegar a ser diferente de lo que son.

Así que los recorridos de estos sujetos antes de llegar a la educación formal, así como las dificultades encontradas para la permanencia, son cuestiones siempre presentes entre los que trabajan e investigan a los adultos en el ámbito educativo. Muchos de ellos, por sus aprendizajes in progress no tienen el dominio del lenguaje que les permita escribir sus propios textos, pero son capaces de desarrollar una narrativa oral sobre sí mismo que puede y debe ser explotada por aquellos que se dedican a intentar entender quiénes son estos que acceden a la educación en una edad mayor que la esperada por los estándares de la escuela.
Mucho de esta historia, que es personal y ubicada dentro de un marco narrativo de un relato de sí mismo, es el registro de estos trayectos de vida y formación. Por eso, saber oír a alguien que está en esta condición es algo que los investigadores de la educación de adultos deben tener en cuenta. La elección de una modalidad o tipo de instrumento de recolección de una narrativa, o de fragmento, o respuesta, puede significar el buen o mal caminar de la recolección de los datos, del análisis y de la investigación en sentido estricto.

Esta observación, demasiado obvia, podría no ser necesaria si la elección del instrumento fuera de hecho una elección. Los investigadores suelen reproducir en sus prácticas muchos los paradigmas de trabajo de sus grupos de investigación, de sus asesores, de las instituciones a la cual están asociados y, muchas veces, la moda ${ }^{6}$. Elegir es un serio problema para la investigación. Y cuando este problema se convierte en la principal fuente algunas veces, la única - de informaciones de lo que piensa un determinado grupo, esta elección deja de ser solamente un problema de investigación para convertirse en un serio desafío que debe ser enfrentado, ya que la historia del otro depende del buen registro para que esta exista. Si no se realiza un buen registro quizás no se tenga material suficiente para empezar una investigación, como mucho se tendrá un material representativo de lo que piensa el entrevistado.

La investigación a través de entrevistas con Adultos guarda la sorpresa de lo inesperado y su naturaleza no puede cerrarse a una única tipología de entrevista. Desde lo presentado en este artículo, podemos trabajar con una tipología de entrevista sin saber si esto es lo más adecuado al perfil del entrevistado. Por ejemplo, desde una mirada etimológica, una entrevista a élites o a expertos sería muy inadecuada para el trabajo con adultos en proceso educativo, ya que no dice nada sobre el tránsito y las posibilidades de actuación social del entrevistado. Muchos adultos en proceso educativo, a veces en procesos de alfabetización, tienen un papel de liderazgo en sus comunidades, en sindicatos, o tuvieron papeles sociales destacados en su sociedad de origen, en el caso de los inmigrantes. Por esto, pensar en su condición de experto o miembro de una élite no puede cerrarse en perspectivas basadas en creencias sobre el entrevistado. Por otra parte la entrevista, sin otros mecanismos de recolección y análisis, no es suficiente para decir algo sobre la complejidad de un adulto que llega a un espacio educativo. 
Por tanto, solamente los modelos de entrevistas no son suficiente, como el propio instrumento de recolección no lo es tampoco, para informar un mínimo acerca de la vida tras los floodlight de la investigación. Es necesario combinar, mixturar, completar para que la entrevista deje de ser excesivamente seca para entrar en un movimiento analítico, a partir del cual sea una propulsora, no un fin.

Por otro lado, desde una dimensión política de la educación de adultos, impregnada desde Freire (1974) - al menos esto es una realidad en América Latina -, los adultos de esta modalidad de educación están inmersos en una educación que intenta transformar su realidad a través de la emancipación y de su participación en las dimensiones micrológicas de la política social. Esto genera en contextos de alfabetización, donde no se esperaría una capacidad crítica, discursos complejos sobre la realidad, donde la consciencia forma parte del aprendizaje tanto en lo referido a las letras, las palabras y los textos. De ahí, que entrevistar a esta gente sea un ejercicio de confrontación de prejuicios sociales, académicos y de representación con uno mismo, moviéndonos dinámicamente más allá de los espacios determinados por la sociedad.

Por último, las discusiones presentadas sobre los aspectos teóricos y metodológicos, referidos al uso de las entrevistas narrativas en el contexto de la investigación cualitativa y su utilidad en los estudios en el campo sobre Educación de Adultos, abren múltiples posibilidades para otras reflexiones significativas sobre las historias individuales y colectivas de las personas en formación, especialmente en lo que se refiere a los modos en que los adultos viven sus vidas, narran sus historias de aprendizajes y sus procesos de inserción y re-inserción social. Resaltamos que el acto de narrar se inscribe en la temporalidad y en la reflexividad biográficas, los cuales generan procesos de potenciación, de resistencia y resiliencia sobre la vida y sus diferentes manifestaciones cotidianas, implicando en disposiciones sociales y políticas a los adultos en relación con sus proyectos de vida-formación.

\section{Notas}

1 No ignoramos todos los cambios de orden epistemológico y metodológico operado por los dispositivos de "más allá", que vienen con los prefijos "post"; y de la crítica a estos movimientos en sus bases de producción conceptual, sobre todo en los campos anti-disciplinarios como los Estudios Culturales y de los múltiples "giros' lingüísticos, culturales, narrativos, afectivos y performativos (KLINGER, 2014; WULF, 2013; ARFUCH, 2010). Lo presentado no es una reducción, pero una opción de escritura.

2 Aquí llamamos técnica, pero la Hermenéutica puede ser entendida como un método y aun mismo como discurso (SOUZA, 2011).

3 Esta mirada ha producido un cambio significativo en los estudios desarrollados por las Ciencias Humanas en el siglo XX e XXI, sobre todo a partir de las miradas de las culturas subalternas, en el abordaje de SPIVAK (2010) o en los perspectivismo amerindio, de Castro (2015).

4 Resaltamos aquí algunas aproximaciones que hemos implementado con entrevistas narrativas desde el Grupo de Investigación (Auto)Biografia, Formação e História Oral, de la Universidade do Estado da Bahia - GRAFHO/UNEB, en Brasil, especialmente: en lo que se refiere a las entrevistas con destacados educadores bahianos (SOUZA, E.C, 2011, 2016; SOUZA; ALMEIDA, 2012); con profesoras de aulas multigrado (también também conocidas como Escuelas Nuevas, en español, sobre todo en Sudamérica) de escuelas rurales del estado de Bahía (SOUZA, 2012); actualmente, con narrativas de enfermos crónicos y procesos de aprendizajes con la docencia, cuando narran sobre sus experiencias con la enfermedad como forma de empoderamiento y reflexividad biográfica sobre la enfermedad (SOUZA, 2016a). Tales experiencias llevadas a cabo con entrevistas narrativas han dialogado con contribuciones teóricas de DeloryMomberger (2012), Jovchelovitch y Bauer (2002), Schütze (2010) y, más precisamente, con las contribuciones de Appel (2005), en lo que se refiere al trabajo con entrevista autobiográfica narrativa realizada por este autor, de acuerdo con las bases teóricas y metodológicas desarrolladas por Fritz Schütze (1981).

5 En diferentes realidades culturales se adoptan terminologías distintas para referirse a este concepto: Educación de Jóvenes y Adultos en Brasil, Educación de Personas Jóvenes y Adultas en los países hispanohablantes de América Latina, son ejemplos de una variación terminológica de la modalidad. Sus variaciones dicen más de los contextos en los cuales se producen que de cambios conceptuales.

6 Sobre las modas y la reproducción en la producción científica es interesante leer el artículo 
de Leslie (2016). Bourdieu y Passeron (1976) siguen siendo una referencia sobre la reproducción en las sociedades capitalistas y el papel que la escuela desempeña en este proceso. Se pueden adoptar las discusiones presentadas ahí para una crítica a los procesos reproductivos sin reflexión en la investigación científica producida en las universidades.

\section{Referencias}

APPEL, M.. La entrevista autobiográfica narrativa: Fundamentos teóricos y la praxis del análisis mostrada a partir del estudio de caso sobre el cambio cultural de los Otomíes en México. Forum: Qualitative Social Research, 6(2), 2005. Disponível em

http://nbnresolving.de/urn:nbn:de:0114fqs0502160. Acesso em 15/08/2015.

ARFUCH, L. El Espacio Biográfico: dilemas de la subjetividad contemporánea. Buenos Aires: Fondo de Cultura Económica, 2010.

ATKINSON, R. The life story interview. Qualitative Research Methods Series. Thousand Oaks, CA: Sage, 1998.

BOURDIEU, P. A distinção: crítica social do julgamento. Tradução de Daniela Kern; Guilherme J. F. Teixeira. São Paulo: Edusp; Porto Alegre: Zouk, 2007.

BOURDIEU, P.; PASSERON, J-C. A Reprodução. Tradução de Reynaldo Bairão. Rio de Janeiro: Francisco Alves, 1976.

CASTRO, E. V. Metafísicas Canibais: elementos para uma antropologia pós-estrutural. São Paulo: Cosac Naify, 2015.

DELORY-MOMBERGER, C. Abordagens metodológicas na pesquisa biográfica. Revista Brasileira de Educação, vol.17, no. 51, p. 523-536, dez. 2012. Disponível em http://www.scielo.br/pdf/rbedu/v17n51/02.pdf. Acesso em 10/08/2014.

DENZIN, N. K. y LINCOLN, Y. S. Manual de investigación cualitativa: el campo de la investigación cualitativa. Barcelona: Gedisa, 2012.
DEXTER, L.A. Elite and specialized interviewing. Evanston: Northwestern University Press, 1970.

FLICK, U. Introducción a la investigación cualitativa. Madrid: Ediciones Morata, 2012.

FLICK, U. Uma introdução à pesquisa qualitativa. Tradução de Sãndra Netz. Porto Alegre: Bookman, 2004.

FREIRE, P. Educação como Prática da Liberdade. Rio de Janeiro: Paz e Terra, 1974.

GOETZ, J. P.; LECOMPTE, M. D. Etnografía y diseño cualitativo en investigación educativa. Madrid: Ediciones Morata, 1988.

GONZÁLEZ-MONTEAGUDO, J. La entrevista en Historia oral e Historias de vida: Teoría, método y subjetividad. In: BENADIBA, L. (Comp.):

Historial Oral: Fundamentos metodológicos para reconstruir el pasado desde la diversidad. Rosario, Argentina: Suramérica Ediciones, 2010, p. 21-38.

HERMANNS, H. Narratives Interviews. In: FLICK, U.; KARDORFF, E.V.; KEUPP, H.;

ROSENSTIEL, L.V.; WOLFF, S. (Eds.).

Handbuch Qualitative Sozialforschung. Munich:

Psychologie Verlags Union, 1995, p. 182-185.

HUSSERL, E. La Idea de la Fenomenología: cinco lecciones. Traducción de Miguel García-Baró. Madrid: Ediciones FCE, 1982.

JOVCHELOVITCH, S.; BAUER, M.W. Entrevista narrativa. In: BAUER, M. W. GASKELL, G. Pesquisa qualitativa com texto, imagem e som. Tradução: Pedrinho Guareschi. Petrópolis, RJ: Vozes, 2002.

KLINGER, D. Literatura e Ética: da forma para a força. Rio de Janeiro: Rocco, 2014.

KVALE, S. Las entrevistas en investigación cualitativa. Madrid. Ediciones Morata, 2011.

LESLIE, I. The Sugar Conspiracy. The Guardian. London. 7 de abril de 2016. Disponível em: https://www.theguardian.com/society/2016/apr/07/t he-sugar-conspiracy-robert-lustig-john-yudkin. Acesso em 23/06/2016.

MUYLAERT, C.J.; SARUBBI JR,V.; GALLO, P. 
R.; ROLIM NETO, M.L.; REIS, A. O. A.

Entrevistas narrativas: um importante recurso em pesquisa qualitativa. Revista da Escola de Enfermagem da USP, vol.48, número especial 2, p.184-189, 2014. Disponível em:

http://www.scielo.br/pdf/reeusp/v48nspe2/pt_00806234-reeusp-48-nspe2-00184.pdf. Acesso em 25/06/2016.

OCÁDIZ, A. E. La Etnometodología, una alternativa relegada de la educación. In: Revista Iberoamericana para la Investigación y el Desarrollo Educativo, vol. 6, n. 12, 2016. Disponível em: http://www.ride.org.mx/index.php/RIDE/article/vie w/193/848. Acesso em 26/10/2016.

RICOEUR, P. Teoria da interpretação: o discurso e o excesso de significação. Tradução de Artur Morão. Lisboa: Edições 70, 2011.

RIESMAN, D. Abundance for What? And other Essays. Garden City, N.Y.: Doubleday, 1964.

SANTOS, B. S. Um discurso sobre as Ciências. São Paulo: Cortez, 2006.

SCHLEIERMACHER, F.D.E. Hermenêutica: arte e técnica de interpretação. Tradução de Celso Reni Braida. Petrópolís: Editora Vozes, 2001

SCHMIDT, L. K. Hermenêutica. Tradução de Fábio Ribeiro. Petrópolis: Editoria Vozes, 2012.

SCHÜTZE, F. Pesquisa biográfica e entrevista narrativa. In.: WELLER, W., PFAFF, N. (Orgs.). Metodologias da pesquisa qualitativa em Educação. Petrópolis: Vozes, 2010, p. 210-222.

SCHÜTZE, F. Pressure and Guilt: war experiences of a young german soldier and their biographical implication. International Sociology, vol. 7, n. 2, 1992, p. 187-208.

SCHÜTZE, F.. Prozessstrukturen des Lebensablaufs. In: MATTHES, J.; PFEIFENBERGER, A. \& STOSBERG, M. (Eds.). Biographie in handlungswissenschaftlicher Perspektive. Nürnberg: Sozialwissenschaftliches Forschungszentrum der Universität Erlangen Nürnberg 1981, p. 67-156.

SOUZA, E. C. de. Biografar-se e empoderar-se: entrevista autobiográfica-narrativa e percursos de formação da professora Dilza Atta. In.:

ABRAHÃO, M. H. M. B. (ORg.). Destacados educadores brasileiros: suas histórias, nossa história. Porto Alegre: EdiPUCRS, 2016, p. 65-95.

SOUZA, E. C. Existir para resistir: (auto)biografia, narrativas e aprendizagens com a doença. Revista da FAEEBA - Educação e Contemporaneidade, Salvador, v. 25, n. 46, p. 59-74, maio/ago. 2016a. Disponível em http://www.uneb.br/revistadafaeeba/files/2011/05/F AEEBA_n46_web1.pdf. Acesso em 05/10/2016.

SOUZA, E, C. Pontes e muros: pesquisa narrativa e trajetórias (auto)biográficas - o lugar da memória e a memória do lugar na educação rural. In.: SOUZA, E. C.; BRAGANÇA, I. F. S. (Orgs.) Memória, dimensões sócio-históricas e trajetórias de vida. Natal: EDUFRN; Salvador: EDUNEB; Porto Alegre: EdiPUCRS, 2012, p. 33-56. (Coleção Temas Transversais, V. 3)

SOUZA, E. C. de. Territórios das escritas do eu: pensar a profissão - narrar a vida. Educação PUCRS, v. 34, p. 213-220, maio/ago. 2011. Disponível em http://revistaseletronicas.pucrs.br/ojs/index.php/face d/article/view/8707. Acesso em 20/07/2014.

SOUZA, E. C. de; ALMEIDA, J. B. Narrar histórias e contar a vida: memórias cotidianas e histórias de vida de educadores baianos. ABRAHÃO, M. H. M. B. (Org). Pesquisa (auto)biográfica em rede. Natal: EDUFRN; Salvador: EDUNEB; Porto Alegre: EdiPUCRS, 2012, p. 31-49. (Coleção Temas Transversais, V. 8)

SOUZA, R. M. A Inscrição no Mundo: apontamentos sobre História da Leitura, Hermenêutica e Estética da Recepção. Praxis: revista eletrônica de história e cultura, v.5, p.16 27, 2011. Disponível em http://revistas.unijorge.edu.br/praxis/2011/pdf/16_aI nscricaoNoMundo.pdf. Acesso em 25/06/2016.

SPIVAK. G. C. Pode o subalterno falar? Tradução de Sandra Regina Goulart Almeida, Marcos Pereira Feitosa e Andrá Pereira Feitosa. Belo Horizonte: Editora UFMG, 2010.

TUCÍDIDES. Historia de la guerra del Peloponeso. Madrid. Ediciones Akal, 1989. 
VALLÉS, M.S. Entrevistas Cualitativas. Madrid: Centro de Investigaciones Sociológicas, 2009.

WELLER, W.; ZARDO, S. P. Entrevista Narrativa com Especialistas: aportes metodológicos e exemplificação. Revista da FAEEBA - Educação e Contemporaneidade, Salvador, v. 22, n. 40, p. 131143, jul./dez. 2013. Disponível em http://www.revistas.uneb.br/index.php/faeeba/articl e/viewFile/757/530. Acesso em 25/06/2016.
WENGRAF, T. Qualitative Research Interviewing. London: SAGE, 2012.

WITZEL, A. Das Problemzentrierte Interview. In: JÜTTEMANN, G. Qualitative Forschung in der Psychologie. Weinheim: Beltz, 1985, p. 227-255.

WULF, C. Homo Pictor: Imaginação, ritual e aprendizado mimético. Tradução de Vinicius Spricigo. São Paulo: Hedra, 2013.

\section{Sobre os Autores}

Rafael M. Hernández-Carrera es Doctor Europeo en Educación y Sociedad. Profesor del Departamento de Educación y Psicología Social, adscrito al área de Didáctica y Organización educativa, de la Universidad Pablo de Olavide de Sevilla, España, con docencia en los Grados de Trabajo Social y Educación Social así como en el Máster Oficial en Educación para el Desarrollo, Sensibilización Social y Cultura de Paz. También es profesor del Área de Didáctica de la Facultad de Educación en la Universidad Internacional de la Rioja donde imparte docencia en los Grados de Maestro en Educación Infantil y Primaria y en el Master en Liderazgo, Dirección y gestión de Centros Educativos. Ha sido profesor del Master en Educación de Personas Adultas en Europa de la Universidad de Sevilla.

Rodrigo Matos de Souza é professor do Centro Universitário Jorge Amado (UJ) e da Faculdade São Salvador (FSS). Doutor em Educação e Contemporaneidade pela Universidade do Estado da Bahia, com doutorado sanduíche pela Universidad de Sevilla (PDSE-BEX/CAPES) e pela Universidade de São Paulo (PROCAD/CAPES). Pesquisador do GRAFHO/UNEB e do CULT-EJA/UNEB. É editor-executivo da Revista Brasileira de Educação de Jovens e Adultos e editor-assistente da Revista Brasileira de Pesquisa (Auto)Biográfica. Já esteve como professor visitante na Universidad Católica de Pereira e é professor internacional do doutorado Formación en Diversidad da Universidad de Manizales, ambas na Colômbia.

Elizeu Clementino de Souza é Professor Titular do Programa de Pós-Graduação em Educação e Contemporaneidade, da Universidade do Estado da Bahia (PPGEduC-UNEB). Pesquisador 1D CNPq. Coordenador do Grupo de Pesquisa (Auto)biografia, Formação e História Oral (GRAFHO/UNEB). Editor Geral da Revista da FAEEBA - Educação e Contemporaneidade e Editor Executivo da Revista Brasileira de Pesquisa (Auto)biográfica (RBPAB). Pesquisador associado do Laboratorie EXPERICE (Université de Paris 13-Paris 8). Foi Presidente da Associação Brasileira de Pesquisa (Auto)biográfica (BIOgraph) nas gestões (2008-2010; 20102012 e 2012-2014), atualmente é Tesoureiro da BIOgraph (2014-2016) e foi Secretario Geral (2011-2013) e Diretor Financeiro da ANPEd (2013-2015). Membro do Conselho de Administração da Association Internationale des Histories de Vie en Formation et de Recherche Biographique en Education (ASIHIVIF-RBE).

Recebido em setembro de 2016.

Aprovado em novembro de 2016. 